\title{
Evaluation of rapid methods of identifying group B streptococci
}

\author{
SHEENA A WAITKINS \\ From the Public Health Laboratory, Fazakerley Hospital, Lower Lane, Liverpool L9 7AL, UK
}

SUMMARY Rapid methods of identifying Lancefield group B streptococci were compared to the standard Fuller's extraction method. Such tests as sodium hippurate hydrolysis, bile tolerance, aesculin hydrolysis, pyruvate fermentation, Camp factor, pigmentation, and bacitracin haemolysis were tested on both routine clinical specimens and National Collection of Type Culture strains. The results show that pigmentation on Islams' medium was the most definitive test available rapidly to identify group B streptococci.

Group B streptococci can cause severe illness in both adults and young children. Mhalu ${ }^{12}$ showed that group B organisms were the most commonly isolated $\beta$ haemolytic streptococci from clinical material, which included specimens from septicaemias, urinary tract infections, and pyogenic skin conditions. These streptococci have also become important as a cause of neonatal morbidity and mortality. ${ }^{3}$ The illness may present as an early septicaemic form in the neonate, which rapidly progresses with or without pneumonia and meningitis to death, between 60 and $75 \%$ of these babies dying within 48 hours of the onset of infection. Alternatively, it may occur as a late-onset meningitis, possibly resulting from nosocomal infection. ${ }^{4-7}$ Because of the potential seriousness of group B streptococci, quick and reliable methods of presumptive identification have been developed.

One of these rapid tests is the hydrolysis of sodium hippurate. This depends on the detection of the end products, benzoic acid and glycine, after hydrolysis of hippurate by the enzyme hippuricase. Ayers and Rupp ${ }^{8}$ first showed that ferric chloride would precipitate benzoic acid as the dark brown/ orange ferric benzoate salt, thus detecting the hippuricase positive human group B streptococci. This method requires a long incubation period of four days, and the ferric chloride added must be titrated each time before use so it is of no value as a rapid diagnostic technique. Hwang and Ederer in $1975^{\circ}$ devised a rapid test in which the end product,

Received for publication 28 August 1979 glycine, was detected within 2 hours using ninhydrin, while Edberg and Samuels ${ }^{10}$ overcame the problem by adding a mixture of Rhodamine $B$ and uranyl acetate to a $1 \%$ sodium hippurate brain heart infusion broth, which results in a fluorescent pink colour if the test is positive. Both methods identify group B streptococci very efficiently.

Another rapid identification procedure peculiar to group B organisms makes use of their ability to utilise starch to produce a bright orange pigment when incubated anaerobically. Fallon ${ }^{11}$ originally used Columbia agar and found a $94 \%$ correlation with Lancefield's methods. Islams ${ }^{12}$ increased the starch concentration and used a tryptone yeast extract base, which gave excellent results when compared to standard grouping methods. Similarly, the Camp test is used routinely in the laboratory. This Camp test is performed on sheep blood agar plates when the streptococci are streaked at rightangles to a staphylococcus that produces beta toxin when incubated aerobically. The lytic phenomenon results in a distinct 'arrowhead' of haemolysis pointing towards the staphylococcus if there is a group B streptococcus. ${ }^{13}$ With such a variety of rapid presumptive tests available, the clinical bacteriologist is presented with a bewildering choice for screening potential group B streptococci in a busy routine laboratory.

This paper evaluates the various methods available using a variety of $\beta$ haemolytic and non-haemolytic streptococci. The strains were tested for susceptibility to bacitracin, ${ }^{14}$ growth on aesculin hydrolysis and bile-aesculin agar, ${ }^{15}$ and pyruvate fermentation ${ }^{16}$ as well as full characterisation of Lancefield groups A, B, C, D, and L streptococci. 
Material and methods

ORGANISMS

Both $\beta$ and non-haemolytic streptococci sent to the Streptococcal Reference Laboratory, Colindale, for routine identification were used to evaluate the rapid tests listed below. The organisms included 40 strains of group A, 170 group B, 55 group D (including 45 Strep. faecalis and 10 Strep. faecium), and 7 group L strains. They were grown on $5 \%$ horse blood agar, and both their sensitivity to bacitracin ( $0 \cdot 1 \mathrm{unit} / \mathrm{disc})$ and their haemolysis reactions were recorded.

\section{LANCEFIELD GROUPING}

Antigenic extracts were prepared using the formamide method of Fuller ${ }^{17}$ and specifically detected using gel diffusion.

\section{CO-AGGLUTINATION}

This test was performed as described by Maxted et al. 18

SODIUM HIPPURATE HYDROLYSIS

The hydrolysis of sodium hippurate was determined by the following methods:

\section{Modification of Ayers and Rupp's ${ }^{8}$ method by Hare and Colebrook ${ }^{19}$}

This method detected the precipitation of benzoic acid by $12 \%$ ferric chloride. A positive reaction was indicated by a bright, brick-orange precipitate.

\section{Hwang and Ederer ${ }^{9}$}

In this method the end product of sodium hippurate, hydrolysis-glycine, was detected by ninhydrin. A deep, dark blue coloration of the test solution resulted if a positive reaction occurred.

\section{Edberg and Samuels ${ }^{10}$}

This method depends on the rapid detection of benzoic acid from the hippurate hydrolysis. A mixture of Rhodamine B and uranyl acetate was used to detect the benzoic acid from organisms grown overnight in an infusion broth. A brigh, dark pink, fluorescent colour indicated a positive reaction.

\section{PYRUVATE FERMENTATION}

The method described by Waitkins ${ }^{16}$ was used. Only group D Strep. faecalis ferments pyruvate to a bright yellow end point.

\section{CAMP REACTION}

The production of Camp factor was assayed by the method of Christie et al.13 A toxin-producing staphylococcus was inoculated diagonally on to a sheep blood agar plate, and the test streptococcal strains were streaked at right-angles to it. The plate was then incubated aerobically at $35^{\circ} \mathrm{C}$ overnight; a positive reaction produced a distinct 'arrowhead' 1 to $2 \mathrm{~mm}$ from the staphylococcal streak.

\section{PIGMENT PRODUCTION}

Fallon $^{11}$

Test strains were inoculated on to Columbia agar plates (Oxoid) and incubated anaerobically using Gas Pak (BBL) overnight at $37^{\circ} \mathrm{C}$. A positive reaction resulted in the formation of reddish, pigmented colonies.

\section{Islams $^{12}$}

Islams' medium was used and incubated as above for Fallon's method, a bright orange pigmentation indicating the growth of group B streptococci.

BILE TOLERANCE AND AESCULIN HYDROLYSIS The ditch plate method, as described by Parker and Ball, ${ }^{15}$ was used to determine tolerance to $10 \%$ and $40 \%$ bile.

\section{Results}

The Table presents a summary of results on 287 strains of streptococci tested. Lancefield grouping by both the Fuller's and co-agglutination methods gave excellent unequivocal results and must be considered as the baseline for identification with which all other rapid methods must stand comparison. Unfortunately, group D streptococci could not be tested by co-agglutination methods because the appropriate antiserum was not available. The results of conventional and rapid methods for detecting hippuricase activity with groups A, B, C, D, and L were worthy of particular note. Not only did group B organisms hydrolyse hippurate but a significant number of other groups tested also gave positive reactions, particularly groups $\mathbf{D}$ and $\mathbf{L}$. All sodium hippurate test systems performed equally poorly. Although rapid, this lack of discrepancy seems to invalidate its use.

The results recorded when streptococci were tested for pyruvate fermentation showed that only Strep. faecalis gave a positive reaction while all other streptococci were negative. Similarly, bile tolerance at $40 \%$ and aesculin hydrolysis was predominantly a group $\mathrm{D}$ reaction. Pigment production, on the other hand, was exclusively a group B phenomenon, and only method variation affected the results. Fallon's method (131/170) produced pigment while Islams' medium performed significantly better $(157 / 170,93 \%)$. In no case did non-group B organisms pigment nor did those colonies that were pigmented belong to any other group than B. This test seemed to be a significantly definitive rapid 
Comparison of various rapid methods for identifying group B streptococci

\begin{tabular}{|c|c|c|c|c|c|c|}
\hline \multirow[t]{3}{*}{ Number of strains tested } & \multicolumn{6}{|c|}{ Group } \\
\hline & \multirow{2}{*}{$\begin{array}{l}A \\
(40)\end{array}$} & \multirow{2}{*}{$\begin{array}{l}B \\
(170)\end{array}$} & \multirow{2}{*}{$\begin{array}{l}C \\
\text { (25) }\end{array}$} & \multicolumn{2}{|l|}{$D$} & \multirow{2}{*}{$\begin{array}{l}L \\
\text { (7) }\end{array}$} \\
\hline & & & & $\begin{array}{l}\text { Strep. faecalis } \\
(45)\end{array}$ & $\begin{array}{l}\text { Strep. faecium } \\
\text { (10) }\end{array}$ & \\
\hline \multicolumn{7}{|l|}{ Lancefield } \\
\hline Fuller's method & $100^{*}$ & 100 & 100 & 100 & 100 & 100 \\
\hline Co-agglutination & 100 & 99 & 96 & ND & ND & ND \\
\hline Pyruvate fermentation & $\mathbf{0}$ & $\mathbf{0}$ & 0 & 100 & 0 & $\mathbf{0}$ \\
\hline \multicolumn{7}{|l|}{ Bile tolerance } \\
\hline $10 \%$ & $\mathbf{0}$ & 82 & 4 & 100 & 100 & 0 \\
\hline $40 \%$ & $\mathbf{0}$ & 8 & $\mathbf{0}$ & 100 & 90 & 0 \\
\hline Aesculin hydrolysis & $\mathbf{0}$ & 0 & $\mathbf{0}$ & 100 & 100 & 0 \\
\hline Non-haemolysis & 0 & 10 & 0 & 60 & 90 & 0 \\
\hline B haemolysis & 100 & 90 & 100 & 40 & 10 & 100 \\
\hline Camp test & 0 & 84 & 0 & 0 & 0 & 0 \\
\hline \multicolumn{7}{|l|}{ Sodium hippurate hydrolysis } \\
\hline Hwang and Ederer & 0 & 92 & 16 & 47 & 60 & 43 \\
\hline Edberg and Samuels ${ }^{10}$ & 0 & 98 & 20 & 73 & 80 & 29 \\
\hline Ayers and Rupp ${ }^{8}$ & 0 & 99 & 16 & 67 & 60 & 14 \\
\hline Pigment on Islams' medium & $\mathbf{0}$ & 93 & 0 & 0 & 0 & 0 \\
\hline Pigment on Columbia medium & 0 & 77 & 0 & 0 & 0 & 0 \\
\hline Bacitracin sensitivity & 100 & 7 & 0 & $\mathbf{0}$ & 0 & 100 \\
\hline
\end{tabular}

- Data presented as percentages.

ND = not done.

identification method for group B. The Camp reaction was also useful $(143 / 170,84 \%)$, again definitive, and, although rapid, it did require a great deal of preparation time.

Neither haemolysis nor bacitracin sensitivity could satisfactorily differentiate between Lancefield groups A, L, or, in some cases, B. Although these tests are used routinely in laboratories they are limited to screening procedures only.

\section{Discussion}

The results show that not all rapid methods of identifying streptococci are reliable. If the laboratory were to use sodium hippurate hydrolysis as a means of recognising group B organisms, then serious misidentification would certainly occur. Alternative methods such as bacitracin resistance, although useful, may again confuse the identification, particularly with group D streptococci. Neither sodium hippurate nor bacitracin could be used alone as a presumptive test; elimination of group $D$ could be achieved by utilising the pyruvate fermentation, bile tolerance, and aesculin hydrolysis, but all of these methods increase the workload of the laboratory and are unsatisfactory since they prove the identification of group B by exclusion rather than by positive means. One test that could identify positively is the Camp reaction, since only group $\mathbf{B}$ streptococci are positive, but as a rapid screening procedure it proved to be too time-consuming and labour- intensive to perform. The most straightforward and easy method was the pigmentation of group B on starch medium; both Fallon's and Islams' methods were exclusive to group B, Islams' giving better results than Fallon's medium $(93 \%: 77 \%)$. The inoculation procedure is simple and could be included in primary cultivation from clinical specimens; the bright orange pigmentation is distinctive and easy to recognise. Both Islams' and Fallon's media are easy to prepare and can be stored in the refrigerator, and results can be read after overnight anaerobic incubation. It must be noted, however, that $7 \%$ of group B streptococci did not pigment on Islams' medium and $23 \%$ did not pigment on Fallon's (this seems a little excessive, but the strains tested were referred ones and may behave abnormally). It is, therefore, essential that if either of these tests is used as a screening method then non-pigmented strains should be further tested by serological means.

Procedures for prompt and reliable recognition of group B streptococci in mother or baby at the time of delivery could be a valuable aid and might prevent some neonatal deaths. Such rapid screening tests are available, and this paper has shown that the most reliable and quick method is that of pigment recognition on Islams' differential medium; more strains failed to show the characteristic pigment on Fallon's medium.

I thank the staff of the Division of Hospital Infection, Colindale, London and the Public Health 
Laboratory, Fazakerley Hospital, Liverpool for the strains of streptococci used in these tests.

\section{References}

${ }^{1}$ Mhalu FS. Infection with Streptococcus agalactiae in a London hospital. J Clin Pathol 1976;29:309-12.

2 Mhalu FS. Streptococcus agalactiae in urinary tract infections. Postgrad Med J 1977;53:216-8.

${ }^{3}$ Yow M. Group B streptococci: a serious threat to the neonate. J Amer Med Ass 1974;230:1177-8.

4 Franciosi RA, Knostman JD, Zimmerman RA. Group B streptococcal neonatal and infant infections. J Pediat 1973;82:707-18.

${ }^{5}$ Baker CJ, Barrett FF, Gordon RC, Yow MD. Suppurative meningitis due to streptococci of Lancefield group B: a study of 33 infants. J Pediat 1973; 82:724-9.

${ }^{6}$ Barton LL, Feigin RD, Lins R. Group B beta hemolytic streptococcal meningitis in infants. $J$ Pediat $1973 ; 82: 719-23$.

${ }^{7}$ Hood M, Janney A, Dameron G. Beta hemolytic streptococcus group B associated with problems of the perinatal period. Amer J Obstet Gynec 1961; $82: 809-18$.

${ }^{8}$ Ayers SH, Rupp P. Differentiation of hemolytic streptococci from human and bovine sources by the hydrolysis of sodium hippurate. J Infect Dis 1922; 30:388-99.

${ }^{\ominus}$ Hwang M-N, Ederer GM. Rapid hippurate hydrolysis method for presumptive identification of group B streptococci. J Clin Microbiol 1975;1:114-5.
${ }^{10}$ Edberg SC, Samuels S. Rapid, colourimetric test for the determination of hippurate hydrolysis by group B streptococcus. J Clin Microbiol 1976;3:49-50.

${ }^{11}$ Fallon RJ. The rapid recognition of Lancefield group B haemolytic streptococci. J Clin Pathol 1974;27:902-5.

12 Islams AKMS. Rapid recognition of group B streptococci. Lancet 1977;1:256-7. (Letter.)

${ }^{13}$ Christie R, Atkins NE, Munch-Petersen E. A note on a lytic phenomenon shown by group B streptococci. Aust J Exp Biol Med Sci 1944;22:197-200.

${ }^{14}$ Maxted WR. The use of bacitracin for identifying group A haemolytic streptococci. J Clin Pathol 1953; 6:224-6.

${ }^{15}$ Parker MT, Ball LC. Streptococci and aerococci associated with systemic infection in man. $J$ Med Microbiol 1976;9:275-302.

${ }^{16}$ Waitkins SA. Use of pyruvate fermentation compared with tetrazolium reduction in the differentiation of group D streptococci. J Clin Pathol 1978;31:692-5.

${ }^{17}$ Fuller AT. The formamide method for extraction of polysaccharides from haemolytic streptococci. Brit $J$ Exp Path 1938;19:130-9.

${ }^{18}$ Maxted WR, Efstratiou A, Parker MT. Agglutination grouping of streptococci. Lancet 1976;2:692-3. (Letter.)

${ }^{19}$ Hare R, Colebrook L. The biochemical reactions of haemolytic streptococci from the vagina of febrileand afebrile parturient women. J Pathol 1934;39:429-42.

Requests for reprints to: Dr SA Waitkins, Public Health Laboratory, Fazakerley Hospital, Lower Lane, Liverpool L9 7AL, UK. 